\title{
Metabonomics-based omics study and atherosclerosis
}

\author{
Duo-jiao $\mathrm{Wu}^{1,2}$, Bi-jun Zhu ${ }^{2}$ and Xiang-dong Wang ${ }^{2^{*}}$
}

\begin{abstract}
Atherosclerosis results from dyslipidemia and systemic inflammation, associated with the strong metabolism and interaction between diet and disease. Strategies based on the global profiling of metabolism would be important to define the mechanisms involved in pathological alterations. Metabonomics is the quantitative measurement of the dynamic multiparametric metabolic response of living systems to pathophysiological stimuli or genetic modification. Metabonomics has been used in combination with proteomics and transcriptomics as the part of a systems biology description to understand the genome interaction with the development of atherosclerosis. The present review describes the application of metabonomics to explore the potential role of metabolic disturbances and inflammation in the initiation and development of atherosclerosis. Metabonomics-based omics study offers a new potential for biomarker discovery by disentangling the impacts of diet, environment and lifestyle.
\end{abstract}

Keywords: Metabonomics, metabolomics, atherosclerosis, metabolic disturbances, inflammation

\section{Introduction}

Living systems are dynamic and complex, and their behavior may be hard to predict from the properties of individual parts. Systems biology is the strategy of integrating complex data about the interactions in systems of biological components from diverse experimental sources using interdisciplinary tools and personnel [1]. Atherosclerosis is one of the leading causes responsible for cardiovascular morbidity and mortality, a complicated and multifactorial disease associated with genotypes and environmental factors [2,3]. It has been suggested that lipid and inflammatory component play an important role in the pathogenesis of atherosclerosis. Metabonomics is the quantitative measurement of the dynamic multiparametric metabolic response of living systems to pathophysiological stimuli or genetic modification [4]. It is expected that metabonomics will become a more and more important global systems biology tool. Recently metabonomics has been used in conjunction with proteomics and transcriptomics as part of a systems biology description of cardiovascular disease. It utilizes high-throughput approaches to profile large

\footnotetext{
* Correspondence: xiangdong.wang@telia.com

${ }^{2}$ Biomedical Research Center, Zhongshan Hospital, Fudan University, 180

Fenglin Rd, Shanghai, China

Full list of author information is available at the end of the article
}

numbers of patients as part of epidemiology studies to understand how the genome interacts with the development of atherosclerosis [5]. Various metabolites have been identified as indicators for a variety of diseases $[6,7]$. The concentrations of metabolites often vary in response to therapy or disease stage. Furthermore, the metabolites could be used as biomarkers to carry information about the sites and mechanisms of disease. Metabolites have also been used as predictive model for disease risk, individual susceptibility, or as markers of recovery from an illness [8].

Metabolomics requires the employment of efficient analytical tools simultaneously together with bioinformatics. Nevertheless, there is not a single analytical platform nowadays capable of analyzing the full set of metabolites in a biological sample. Metabonomics, and the related field of metabolomics, uses tools such as liquid chromatography-mass spectrometry (LC-MS) or gas chromatography-mass spectrometer (GC-MS)or capillary electrophoresis and nuclear magnetic resonance (NMR) spectroscopy to analyze chemical components [9]. Jeremy K. Nicholson and John C. Lindon [10] said that the distinction between metabonomics and metabolomics is mainly philosophical, rather than technical. The basic principle of relating chemical patterns to biology is same. In practice, the two terms are often used 
interchangeably. Metabolic disturbances are the key factor in both the initiation and progression of atherosclerosis. There is ample evidence that hypercholesterolemia (that is, elevated plasma levels of low-density lipoprotein (LDL) and very low-density lipoprotein (VLDL) induced by genetic modification or enhanced intake of dietary lipids is a major causative factor in atherogenesis $[11,12]$. Because samples of biological fluids (usually urine or blood) can be collected fairly easily, the time-dependent fluctuations of metabolites that occur in response to disease, drug effects or other stimuli. By using GC-MS or NMR, metabonomics can easily study these changes in real-time way. And metabonomics cuts through the problems by monitoring the global outcome of all the influential factors for example environmental and lifestyle factor, without making assumptions about the effect of any single contribution to that outcome.

Moreover, to compare with proteomics and transcriptomics, metabonomics is rather rapid and economic [13]. Metabonomics is also a high throughput approach used in a large scale of population epidemic study, while transcriptomic studies can be quite costly and proteomic studies relatively time consuming [13].

\section{Data handling of metabonomics in atherosclerosis study}

Multiple analytical techniques and metabolome database are developed in recent years. Spectral processing and post-experimental data analysis are the major tasks in metabonomics studies. While in data analysis, the Principal Components Analysis (PCA), Hierarchical Cluster Analysis (HCA), Soft Independent Modeling of Class Analogy (SIMCA) and Artificial Neural Network (ANN) are the major techniques. The researchers could select them according to the research destination [14]. The data generated in metabonomics usually consist of measurements performed on subjects under various conditions. Several statistical programs are currently available for analysis of both NMR and mass spectrometry data. The first comprehensive software was developed by the Siuzdak laboratory at The Scripps Research Institute in 2006. It is called XCMS, is freely available, has over 20,000 downloads since its inception in 2006 [15], and is one of the most widely cited mass spectrometry-based metabolomics software programs in scientific literature. Other popular metabolomics programs [16-18] for mass spectral analysis are MZmine, MetAlign, MathDAMP, which also compensate for retention time deviation during sample analysis. Although a high-throughput metabolomics approach to atherosclerosis studies brings many advantages, it also brings a danger of generating false-positive associations due to multiple testing and overfitting of data. Application of traditional statistical approaches (e.g., Bonferroni correction) in this setting tends to levy an insurmountable statistical penalty that can obscure biologically relevant associations. Even newer statistical techniques $[19,20]$, such as advanced resampling methods or control of the false discovery rate, do not adequately address the fundamental problem of how to detect subtle but important changes in multiple variables identified in an "omics" approach.

Meanwhile, there are more and more database, e.g., Small Molecule Pathway Database (SMPDB), LIPID Metabolites And Pathways Strategy (LIPID MAPS), Human Metabolome Database (HMDB) to support metabolomics study [21,22]. HMDB [23] is a Web-based bioinformatic/cheminformatic resource with detailed information about human metabolites and metabolic enzymes. It could be used for fields of study including metabolomics, biochemistry, clinical chemistry, biomarker discovery, medicine, nutrition, and general education. Since its first release in 2007, the HMDB has been used to facilitate the research for nearly 100 published studies in metabolomics, clinical biochemistry and systems biology.

\section{Metabonomic strategies to study metabolic disturbances and lipotoxicity}

The global collection of metabolites in a cell or organism is often called the metabolome; this refers to all small molecules that exclude nucleic acids and proteins. There is a new term "Lipidomics" [24], a branch of metabolomics, is a systems-based study of all lipids, the molecules with which they interact, and their function within the cell. Using LC-MS based lipidomics, Clish et al [25] demonstrated altered fatty acid metabolism in 9 weeks apolipoprotein E3-Leiden (ApoE*3) transgenic mice with only mild type I and II atherosclerotic lesions, reflected by an increase in lipid triglycerides and a decrease in lyso-phosphocholine. In an analogous manner, Martin JC, et al [26] gave the hamsters high fat diet to examine the suitability of plasma metabonomics to determine the severity of diet-induced atherosclerosis. They found that VLDL lipids, cholesterol, and N-acetylglycoproteins were the most positively correlated metabolites. These metabolites predicted $89 \%$ of atherogenic variability compared to the $60 \%$ predicted by total plasma cholesterol alone. Which demonstrates plasma metabonomics may be helpful in disease diagnosis of diet-induced atherogenesis by identifing novel potential disease biomarkers (Figure 1 is a simplified workflow for a typical metabonomic experiment). Furthermore, analyzing different kinds of body fluid simultaneously could provide more description of disease. Zhang F, et al [27] collected plasma and urine samples from the disease and control rats for the metabonomic analysis. 12 metabolites in plasma and 8 endogenous metabolites in urine were identified as potential biomarkers for 


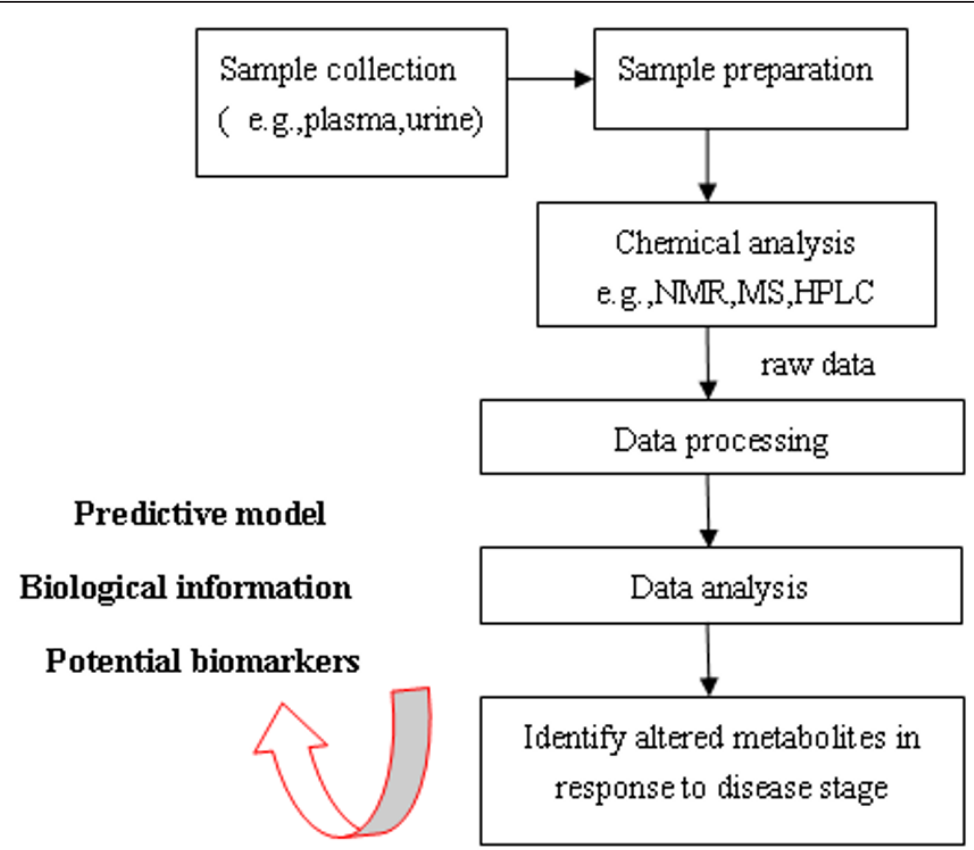

Figure 1 A "typical" and "simplified" workflow for a metabonomic experiment. Samples are collected and extracted for the metabolites measurement. By using combination of techniques and data analysis, metabonomics provides information which could be used to identify potential biomarkers, build predictive models for system biology studies.

atherosclerosis. The altered metabolites suggested abnormal metabolism of phenylalanine, tryptophan, bile acids and amino acids.

Metabolic disturbance of atherosclerosis exist not only in circulation, but also could be found in local vessel. Using 2-dimensional gel electrophoresis and mass spectrometry, researchers identified 79 protein species that were altered during various stages of atherogenesis [28]. Simultaneously by using NMR, they found a decline in alanine and a depletion of the adenosine nucleotide pool in vessels of 10-week-old apolipoprotein E-knockout mice [28]. More importantly, the study demonstrated the power of a combined "-omics" platform. These techniques complemented each other and provided a more comprehensive dataset of protein and metabolite changes during atherogenesis and highlights potential associations of immune-inflammatory responses, oxidative stress, and energy metabolism. The study suggested that vascular cells might respond to hyperlipidemia by metabolizing lipids instead of glucose. Increased fatty acid oxidation would exert a negative feedback on the activity of the pyruvate dehydrogenase complex slowing down glucose metabolism, the main source of energy for the vasculature $[29,30]$. For further elucidation, the researchers observe the effects of attenuating lesion formation. They found it was associated with alterations of reduced form of nicotinamide-adenine dinucleotide phosphate (NADPH) generating malic enzyme, which provides reducing equivalents for lipid synthesis and glutathione recycling, and successful replenishment of the vascular energy pool [28].

Metabonomics also offers a deep insight on the clinical study of atherosclerosis related disease. The plasma of patients with stable carotid atherosclerosis have been fingerprinted with both GC-MS and 1HNMR [31]. 24 metabolites that were significantly modified in the group of atherosclerotic patients and were associated to alterations of the metabolism characteristics of insulin resistance that can be strongly related to the metabolic syndrome. For example, D-glucose, 3-OH-butyrate (3HB) and acetoacetate were increased; citrate, isocitrate, succinate and malate were downregulated. The correlations among the results of GC-MS and $1 \mathrm{H}$ NMR fingerprints can provide complementary information and a deeper insight into the patient state. However, clinical investigation is few, while most studies have focused on the pathophysiological study of atherosclerosis on the animal models. Given the current interest in this field, particularly in drug efficacy assessment and lifestyle and diet interventions, there is urgent needs to enhance clinical metabonomics study [13].

\section{Metabonomic strategies to study inflammation in atherosclerosis}

More and more studies in basic and experimental science have illuminated the role of inflammation and 
the underlying cellular and molecular mechanisms that contribute to atherogenesis. The development of atherosclerosis-induced metabolic perturbations of fatty acids, such as palmitate, stearate, and 1-monolinoleoylglycerol, showed that palmitate significantly contributes to atherosclerosis development via targeting apoptosis and inflammation pathways [32]. Metabolic disturbances in the vasculature stimulate local secretion of inflammatory cytokines. Recent studies implied that the metabolic actions of cytokines such as Interleukin-6 may aim to maintain glucose homeostasis in the smooth muscle cells and contribute to the general adaptation of the vasculature to stress stimuli [33].

Given the strong interaction between metabolic disturbances and inflammation, we would expect metabonomics study should hold substantial promise in defining the mechanism involved in this collection of pathologies. Kleemann and co-workers [34] used a combined metabolomic and transcriptomic study of the liver to investigate the inflammatory component of atherosclerosis that originates in this organ (Figure 2). In highest fat diet (HC) group, atherosclerotic lesions of ApoE*3 Leiden mice was proportional to dietary intake of cholesterol, with pro-inflammation being observed in the liver. To verify whether the switch from metabolic adaptation (with low-cholesterol diets treatment, $\mathrm{LC}$ ) to hepatic inflammatory stress (with $\mathrm{HC}$ treatment) is also reflected at the metabolite level, they performed a comprehensive HPLC/MS-based lipidome analysis (measurement in total of about 300 identified di- and triglycerides, phosphatidylcholines, lysophosphatidylcholines, cholesterol esters) on liver tissue of Con/LC/HC groups and corresponding plasma samples. The clusters of the Con and LC groups overlapped partly, demonstrating that the Con and LC groups have a similar intrahepatic lipid pattern. Which indicates that the metabolic adjustments of genes in the LC group were effective and enabled the liver to adjust to moderate dietary stress. The HC cluster has no overlap with the Con group, showing that the switch to a proinflammatory liver gene expression profile is accompanied by development of a new metabolic hepatic state, which differs significantly from the Con group. Furthermore, while the LC induced transcriptional changes that appeared protective, predominately controlled by sterol regulatory element binding protein (SREBP) 1 and SREBP2, specific $\beta 1$ glycoprotein (SP-1), retinoid $\times$ receptor (RXR) and peroxisome proliferator activated receptor- $\alpha$ (PPAR $\alpha)$, the high-cholesterol diet not only induced inflammation but also altered lipid metabolism, thus linking dyslipidaemia and inflammation in this animal model.

Metabonomics study has also been used to assess antiinflammatory drug efficiency in atherosclerotic cardiovascular disease such as myocardial infarction (MI). Using metabolomic profiling of the inflammatory lipid mediators, Li N and colleagues [35,36] documented a significant decrease in epoxyeicosatrienoic acids/

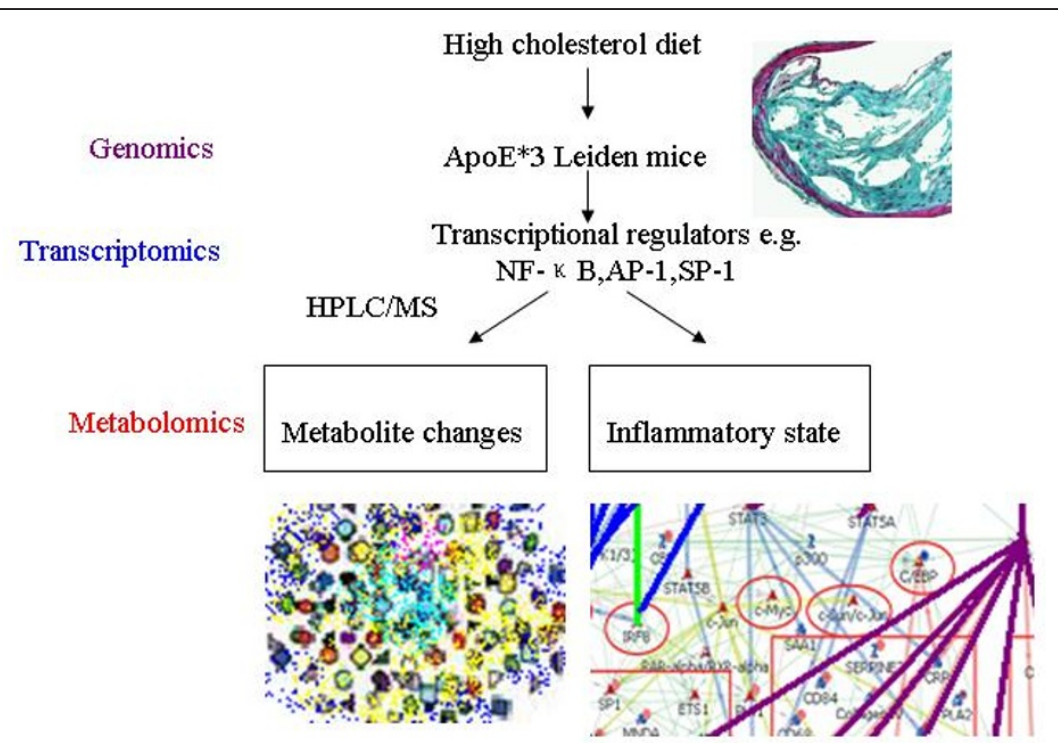

Figure 2 Combined study of transcriptomics and metabolomics in atherosclerosis and liver inflammation induced by high fat diet in ApoE*3 Leiden mice[34]. ApoE*3Leiden mice were treated with high cholesterol diets (HC), scored early atherosclerosis and profiled the pathophysiological state of the liver by using transcriptomics and metabolomics techniques. In HC group, the livers of mice switched from a resilient state to an inflammatory, pro-atherosclerotic state and developed atherosclerosis. HC-evoked changes were regulated by transcriptional master regulators. These regulators control both lipid metabolism and inflammation, and thereby link the two processes. 
dihydroxyeicosatrienoic acids ratio in MI model, which predicted a heightened inflammatory state. Treatment with soluble epoxide hydrolase ( $\mathrm{sEH}$ ) inhibitors caused altered pattern of lipid mediators from inflammation towards resolution. Meanwhile, the oxylipin profiling showed a significant parallel to the changes of inflammatory cytokines in the model. Although few studies are available, metabolomics techniques provide evidence for new therapeutic potentials of cardiovascular disease.

\section{Conclusions}

As the field of metabonomics advances, the ways in which metabolites affect atherosclerotic states will become clearer, and prevention and treatment of this process will become more focused. The integration of metabonomics with genetics, proteomics, and transcriptomics would provide a systems biology description of atherosclerotic cardiovascular diseases. A major challenge in the future will be the bioinformatics side of metabonomics. For systems biology, the integration of multi-level Omics profiles (also across species) is considered as central element $[37,38]$. Traditional statistical methods for the study of static Omics datasets are of limited relevance and new methods are required.

\section{Acknowledgements}

This work was supported by grants from National Nature Science Foundation of China (NSF30670911), the programs of Science and Technology Commission of Shanghai Municipality (03JC14085, 08PJ1402900, 08DZ2293104, 074119634, 09DZ2260300,08410701300 and 09540702600), Key Projects in the National Science \& Technology Pillar Program in the Eleventh Five-year Plan Period (2008BAl60B04), Fudan University and Zhongshan Hospital Grant for Distinguished Professor, and Shanghai Leading Academic Discipline Project (T0206, B115).

\section{Author details}

1'Shanghai Key Laboratory of Organ Transplantation, Zhongshan Hospital, Fudan University, 180 Fenglin Rd, Shanghai, China. ${ }^{2}$ Biomedical Research Center, Zhongshan Hospital, Fudan University, 180 Fenglin Rd, Shanghai, China.

\section{Authors' contributions}

DJW and BJZ drafted the manuscript. XDW designed and helped to revise the manuscript. All authors read and approved the final manuscript.

\section{Competing interests}

The authors declare that they have no competing interests.

Received: 3 October 2010 Accepted: 31 October 2011 Published: 31 October 2011

\section{References}

1. Kitano H: Systems biology: a brief overview. Science 2002, 295:1662-1664

2. Ambrose JA, Srikanth S: Vulnerable plaques and patients: improving prediction of future coronary events. American Journal of Medicine 2010, 123:10-16.

3. Naghavi $M$, Libby $P$, Falk E: From vulnerable plaque to vulnerable patient: a call for new definitions and risk assessment strategies: Part II. Circulation 2003, 108:1772-1778.

4. Nicholson JK: Global systems biology, personalized medicine and molecular epidemiology. Mol Syst Biol 2006, 2:1-6.
5. Lewis GD, Asnani A, Gerszten RE: Application of metabolomics to cardiovascular biomarker and pathway discovery. Journal of the American College of Cardiology 2008, 52:117-123.

6. Mimmi MC, Picotti P, Corazza A, Betto E: High-performance metabolic marker assessment in breast cancer tissue by mass spectrometry. Clin Chem Lab Med 2011, 49:317-324.

7. Patterson AD, Bonzo JA, Li F, Krausz KW, Eichler GS, Aslam S: Metabolomics Reveals Attenuation of the SLC6A20 Kidney Transporter in Nonhuman Primate and Mouse Models of Type 2 Diabetes Mellitus. J Biol Chem 2011, 286:19511-19522

8. Cavill R, Keun HC, Holmes E, Lindon JC, Nicholson JK, Ebbels TM: Genetic algorithms for simultaneous variable and sample selection in metabonomics. Bioinformatics 2008, 25:112-118.

9. Yang J, Song SL, Castro-Perez J, Plumb RS, Xu GW: Metabonomics and its Applications. Sheng Wu Gong Cheng Xue Bao 2005, 21:1-5.

10. Nicholson JK, Lindon JC: Systems biology metabonomics. Nature 2008 455:1054-1056

11. Chen CL, Liu IH, Fliesler SJ, Han X, Huang SS, Huang JS: Cholesterol suppresses cellular TGF-beta responsiveness: implications in atherogenesis. Journal of Cell Science 2007, 120:3509-3521.

12. Fessler MB, Rudel LL, Brown JM: Toll-like receptor signaling links dietary fatty acids to the metabolic syndrome. Current Opinion in Lipidology 2009, 20:379-385.

13. Lindon CJohn, Nicholson KJeremy, Holmes Elaine: The handbook of metabonomics and metabolomics 2006. Elsevier Science.

14. Dieterle F, Riefke B, Schlotterbeck G, Ross A, Senn H, Amberg A: NMR and MS methods for metabonomics. Methods Mol Biol 2011, 691:385-415.

15. Smith CA, Want EJ, O'Maille G, Abagyan R, Siuzdak G: XCMS: processing mass spectrometry data for metabolite profiling using nonlinear peak alignment, matching, and identification. Anal Chem 2006, 78:779-787.

16. Katajamaa M, Miettinen J, Oresic M: MZmine: toolbox for processing and visualization of mass spectrometry based molecular profile data. Bioinformatics 2006, 22:634-636.

17. Lommen A: MetAlign: interface-driven, versatile metabolomics tool for hyphenated full-scan mass spectrometry data processing. Anal Chem 2009, 81:3079-3086.

18. Baran R, Kochi H, Saito N, Suematsu M, Soga T, Nishioka T, Robert M, Tomita M: MathDAMP: a package for differential analysis of metabolite profiles. BMC Bioinformatics 2006, 7:530

19. Storey JD, Tibshirani R: Statistical significance for genomewide studies. Proc Natl Acad Sci USA 2003, 100:9440-9445.

20. Trygg J, Holmes E, Lundstedt T: Chemometrics in metabonomics. $J$ Proteome Res 2007, 6:469-479.

21. Frolkis A, Knox C, Lim E: SMPDB: The Small Molecule Pathway Database. Nucleic Acids Research 2010, 38:D480-487.

22. Wishart DS, Knox C, Guo AC: HMDB: a knowledgebase for the human metabolome. Nucleic Acids Research 2009, 37:D603-610.

23. Wishart DS: Human Metabolome Database: completing the 'human parts list'. Pharmacogenomics 2007, 8:683-686.

24. Watson AD: Lipidomics: a global approach to lipid analysis in biological systems. Journal of Lipid Research 2006, 47:2101-2111.

25. Clish $C B$, Davidov $E$, Oresic M: Integrative biological analysis of the APOE*3-leiden transgenic mouse. OMICS 2004, 8:3-13.

26. Martin JC, Canlet C, Delplanque B: $1 \mathrm{H}$ NMR metabonomics can differentiate the early atherogenic effect of dairy products in hyperlipidemic hamsters. Atherosclerosis 2009, 206:127-133.

27. Zhang F, Jia Z, Gao P: Metabonomics study of atherosclerosis rats by ultra fast liquid chromatography coupled with ion trap-time of flight mass spectrometry. Talanta 2009, 79:836-844.

28. Mayr M, Chung YL, Mayr U: Proteomic and metabolomic analyses of atherosclerotic vessels from apolipoprotein E-deficient mice reveal alterations in inflammation, oxidative stress, and energy metabolism. Arteriosclerosis, Thrombosis, and Vascular Biology 2005, 25:2135-2142.

29. Bryson JM, Cooney GJ, Wensley VR, Phuyal JL, Caterson ID: The effects of the inhibition of fatty acid oxidation on pyruvate dehydrogenase complex activity in tissues of lean and obese mice. International journal of obesity and related metabolic disorders 1996, 20:738-744.

30. Lynch RM, Paul RJ: Compartmentation of glycolytic and glycogenolytic metabolism in vascular smooth muscle. Science 1983, 222:1344-1346. 
31. Teul J, Rupérez FJ, Garcia A: Improving metabolite knowledge in stable atherosclerosis patients by association and correlation of GC-MS and $1 \mathrm{H}$ NMR fingerprints. Journal of Proteome Research 2009, 8:5580-55896.

32. Chen X, Liu L, Palacios G, Gao J, Zhang N, Li G, Lu J, Song T, Zhang Y, Lv H: Plasma metabolomics reveals biomarkers of the atherosclerosis. Sep Sci 2010, 33:2776-2783.

33. Febbraio MA, Hiscock N, Sacchetti M, Fischer CP, Pedersen BK: Interleukin-6 is a novel factor mediating glucose homeostasis during skeletal muscle contraction. Diabetes 2004, 53:1643-1648.

34. Kleemann $R$, Verschuren $L$, van Erk MJ: Atherosclerosis and liver inflammation induced by increased dietary cholesterol intake: a combined transcriptomics and metabolomics analysis. Genome Biology 2007, 8:R200.

35. Li N, Liu JY, Timofeyev V: Beneficial effects of soluble epoxide hydrolase inhibitors in myocardial infarction model: Insight gained using metabolomic approaches. Journal of Molecular and Cellular Cardiology 2009, 47:835-845.

36. Liu JY, Yang J, Inceoglu B: Inhibition of soluble epoxide hydrolase enhances the anti-inflammatory effects of aspirin and 5-lipoxygenase activation protein inhibitor in a murine model. Biochemical Pharmacology 2010, 79:880-887.

37. Buschow SI, Lasonder E, van Deutekom HW: Dominant processes during human dendritic cell maturation revealed by integration of proteome and transcriptome at the pathway level. Journal of Proteome Research 2010, 9:1727-1737.

38. Nibbe RK, Koyutürk M, Chance MR: An integrative-omics approach to identify functional sub-networks in human colorectal cancer. PLOS Computational Biology 2010, 6:1000639.

doi:10.1186/2043-9113-1-30

Cite this article as: Wu et al:: Metabonomics-based omics study and atherosclerosis. Journal of Clinical Bioinformatics 2011 1:30.

\section{Submit your next manuscript to BioMed Central and take full advantage of:}

- Convenient online submission

- Thorough peer review

- No space constraints or color figure charges

- Immediate publication on acceptance

- Inclusion in PubMed, CAS, Scopus and Google Scholar

- Research which is freely available for redistribution

Submit your manuscript at www.biomedcentral.com/submit 